Биклиогра јические соилки

I. К О р н е у х Н.П. Экстремальнне задачи теории приблихения И., I976.

2. Корне ч ук Н.П.. Лигун А.А., Доронин В.Г. Aппроксимация о ограничениями. Kиел, I>82.

3. К О р н И у у К Н.Іl. Экстремальные эначения функционалов $и$ наклучшее приближение на классах периодичөских функцй // И9у. АН СССР. Сер. 探ематика. I97I. T.35, I.

4. А л Cер. 中атекатика, механика и астт номия. 1966. Bun. 2, * 7.

5, С т о р ч а а В. . . Об гтклонении доманнх в метрике $L_{p} / /$ Maт. за.детки. I969. T.5, I.

6. С то рч в В В.Ф.. Л и г у н А.А. Об отклонении некоторых интерполяционных оплаһнов в метриках C и Lp // Теория прнбиижея: яя Функиия и её приложения. Киев, I974.

7. 耳 и $г$ н A.A., $M$ а д Функиия, полученних осреднением кусочно . інеиных // Изв. вузов. Cep. 抽ематика. I977. I.

JIK 5I7. 5

\title{
A.A. Wymeraxo
}

О ВЫБОРЕ УЗЛОВ ДЛП ЛОКАЛЬНЫХ СМЕШАННЫХ ТПЛАЯНОВ

Пусть $\delta_{\mu}=\left\{0=t_{0}<t_{1}<\cdots: t_{\mu}=1\right\}-$ фиксированное раябненив отpesra $[0,1]$ тakoe, 4то $t_{i}=1-t_{\mu-l}(i=\overline{0, \mu})$.

Полохим $r=1,6, \ldots, k=1, r, \quad \mu=\mu_{r, k}=|r+1-2 k|+1$.

для $x \in C^{r-k}$ покальнын сплаћнон $\ell_{r, k}(x, \delta \mu, t)$ назовём функиир, однозначно опредөлярщуося уоловияки

$$
\ell_{r, k}^{(v)}\left(x, \delta_{\mu}, i\right)=x^{(v)}(i) \quad(v=\overline{0, r-k} ; i=0,1)
$$

и при $k \leq(r+1) ; 2:$

I) на колдом интеріале $\left(t_{i-1,} t_{i}\right)(i=\overline{1, \mu})$ зна являетоя адгебраячеоким многочленок отепени не внше $r$;

2) $l_{r, k}\left(x, \delta_{\mu}\right) \in C^{r-1}$

и при $k \geqslant(r+1) / 2$ :

I) $\operatorname{lr,k}\left(x, \delta_{\mu}\right)$ на $[0,1]$ - влгебракческии многочден степени $\leqslant r$ : 
$-72-$

2) $l_{r, k}\left(x, \delta \mu, t_{i}\right)=x\left(t_{i}\right)(i=\overline{0, \mu})$.

Череs $C^{r, s}$ обозначим мнотество всех функинд $f(x, y)$. имеvщих хвадрате $D=[0,1] \times[0,1]$ непрерывнне частнне проияводнне $f^{(i, j)}(x, y)(j=\overline{0, s} ; i=0, r)$ и $L_{p}^{r, s}=L_{p}^{r, s}(D)(p \in[1, \infty])$.

Пусть Ad. $p>0$

$$
\|f\|_{P}=\|f\|_{p(D)}=\left(\iiint_{D}|f(x, y)|^{P} d x d y\right)^{1 / p} .
$$

Paoomorpme xлетки $D_{i}=\left[\alpha_{i}, \beta_{i}\right] \times\left[a_{i}, b_{i}\right]$ и пуоть $h_{i}=\beta_{i}-\alpha_{i}$, $H_{i}=B_{i}-r$.

CrähH

$$
\begin{aligned}
& \operatorname{Pr,k}_{r, x}(f, y)=l_{r, k}\left(f(\cdot, y), S_{\mu},\left(x-\alpha_{i}\right) / h_{i}\right)+\ldots \\
& +l_{r, k}\left(f(x, \cdot), \delta_{\mu},\left(y-a_{i}\right) / H_{i}\right)-l_{r, k}\left(l_{r, k}\left(f(\cdot, \cdots), \delta_{\mu},\left(\cdots a_{i}\right) / H_{i}\right),\right. \\
& \left.\quad \delta_{\mu},\left(x-\alpha_{i}\right) / h_{i}\right)
\end{aligned}
$$

на: рвём омешанным докадьным сплайном. Ясно, Ч Ј на кахдоя клетхе $D_{i}$ он будет опредедяться независимо.

Нетрудно эидеть, что имешаннне оплайн, определённне не на кдеткал, а на решётках, не всякур фу! :цив могут хор до прнбликать, например, өсли $f(x, y)=\varphi(x-y)$ такова, что $\varphi^{(r, r)}(0)$ o."дественно больше $\varphi^{(r, r)}(t)\left(\left.\right|^{\prime} \mid>\delta>0\right)$, то смеганния оплаян, опре дедённй нш рехётках, будет пхохо прибдихать, ках би ни виоирахась pođërka.

Заметия, что омешаннне опдаһнн (или как их наянвадт "бдендингоплайн", ихи тезорное произведение одномерннх овнаинов ) рассматриваттся довольно часто [I, 2].

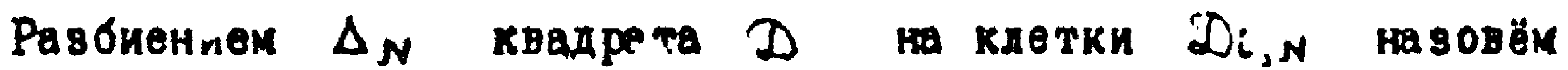
.०бор прямоугольников $\left\{D_{i, N}\right\}_{i=1}^{N}=\left\{\left[\alpha_{i}, \beta_{i}\right] \times\left[a_{i}, b_{i}\right]\right\}_{i=1}^{N}$ co оторонами, паралдедьными координатным осям, таких, что

$$
D_{i, N} \bigcap_{i \neq j} D_{j, N}=\varnothing, \quad \bigcup_{i=1}^{N} D_{i, N}=D \text {. }
$$

Смешанным лохальным опдайлм $P\left(f, \Delta_{N}, x, y\right)$ назовём сметан.

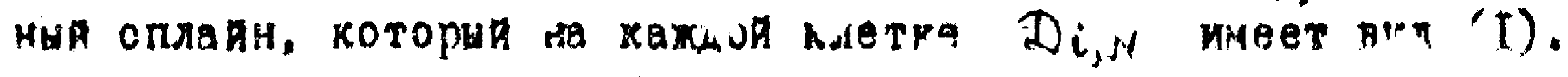

Ph..двм некоторне осавняения: 
$-73-$

$$
\omega(f, t)=\sup \left\{\left|f\left(x_{1}, y_{1}\right)-f\left(x_{2}, y_{2}\right)\right| \mid\left(x_{1}-x_{2}\right)^{2}+\left(y_{1}-y_{2}\right)^{2} \leq t\right\}-
$$

модуль нопреравности фунжиии $f(x, y) \in C^{1,1}$, и пусть

$$
\begin{gathered}
\varphi_{N}(z, x, y)=\varphi_{N, n, m, \alpha, \gamma}(z, x, y)=\left(\mid z_{N}(x, y) \|^{\gamma}\left(\omega^{(r+1, r+1)}, n^{-2}+m^{-2}\right)\right)^{\alpha} \\
\Psi_{N}=\left\|\varphi_{N}(z)\right\|_{\alpha(D)}, \quad \Psi(f)=\left\|f^{(r+1, r+1)}\right\|_{\alpha(D) .}
\end{gathered}
$$

В [3] получвно равенство

$$
x(t)-l_{r, k}(x, t)=\int_{0}^{1} K_{r}(t, u) x^{(r+1)}(u) d u,
$$

.20

$$
K_{r}(t, u)=-\frac{1}{r !}\left\{(u-t)_{+}^{r}-l_{r, r-k+1}\left((\cdot-t)^{r}, u\right)\right\} .
$$

Kpome тоro,

$$
\begin{aligned}
& \int_{0}^{1} X_{r}(t, u) d u=\Theta_{r, k}(t) ; \\
& \int_{0}^{1}\left|K_{r}(t, u)\right| d u=\mathscr{X r}_{r, k}(t),
\end{aligned}
$$

rne

$$
\begin{gathered}
\Theta_{r, k}(t)=\frac{t^{r+1}}{(r+1) !}-l_{r, k}\left(\frac{(\cdot)^{r+1}}{(r+1) !}, t\right) ; \\
x_{r, k}(t)=\frac{1}{r !} \int_{0}^{1}\left|(u-t)_{+}^{r}-l_{r, r-k+1}\left((-t)^{r}, u\right)\right| d u .
\end{gathered}
$$

Tеорема. Пуоть $r, n, m=1,2, \ldots ; N=n \cdot m, k=1, r, p \in[1, \infty]$, $\alpha=\left(r+1+p^{-1}\right)^{-1}, \quad \gamma=(2+\alpha(2 r+3))^{-1}$ и $n, m \rightarrow 0$. Тогд для $\times n-$ бои Функцик $f(x, y) \in L_{\infty}^{n+1, r+1}$ последоватөльночть разбнения $\left\{\Delta_{N}^{*}\right\}_{N=1}^{\infty}=$ $\left.=\left\{\left\{\left\{D_{i, j, N}^{*}\right\}_{i}^{n}\right\}_{j=1}^{m}\right\}_{N=1}^{\infty} \quad\left(D_{i, j, N}^{*}=\left[x_{i-1, j}^{*}, x_{i, j}^{*}\right] \times \Gamma_{j-1}^{*}, y_{j}^{*}\right]\right)$, опрөлеляемая из условия

$$
\int_{0}^{y_{j}^{j}} \int_{0}^{1} \varphi, .\left(z, x, y_{,}^{\prime} d x d y \cdot \frac{j}{m} \psi_{N}^{\alpha}(j=1, m),\right.
$$


$-74-$

$$
\begin{gathered}
\int_{0}^{x_{i, j}^{*}} \int_{y_{j-1}^{*}}^{y_{j}^{*}} \varphi_{N}(z, x, y) d x d y=\frac{i}{N} \Psi_{N}^{\alpha},(i=\overline{1, n} ; j=\overline{1, m}), \\
\left\{z_{N}^{\alpha}\right\}-\infty=1-\text { множество функиия таких, что } \\
\left\|z_{N}-f^{(r+1, r+1)}\right\|_{\infty} \leq \omega^{2 \gamma}\left(f^{(r+1, r+1)}, n^{-2}+r^{-2}\right)
\end{gathered}
$$

будет асимптотичес.и оптимальнои, т.е.

$$
\inf _{\Delta_{N}}\left\|f-P\left(f, \Delta_{N}\right)\right\|_{P}=\left\|f-P f, \Delta_{N}^{*}\right\|_{P}(1+o(1))
$$

причём

$$
\inf _{\Delta_{N}}\left\|f-P\left(f, \Delta_{N}\right)\right\|_{P}=\frac{\|\left(\omega_{r, r} \|_{P}^{2}\right.}{N^{r+1}} \psi(f)+0\left(\frac{1}{N^{r+1}}\right) .
$$

Кроме того, если $f_{\infty}^{(r+1, r+1)}(x, y)$ не имеет нулеи на $D$, то последовательность $\left\{\Delta_{N}^{*}\right\}_{N=1}^{\infty}$, определяемая из равенотв

$$
\begin{aligned}
& \int_{0}^{y_{j}^{*}} \int_{0}^{1}\left|z_{N}(x, j)\right|^{\alpha} d x d y=\frac{j}{m}\left\|z_{N}\right\|_{\alpha}^{\alpha} ; \\
& \int_{0}^{x_{i, j}^{*}} \int_{y_{j-1}^{*}}^{y_{j}^{*}}\left|z_{N}(x, y)\right|^{\alpha} d x d y=\frac{i}{N}\left\|z_{N}\right\|_{\alpha}^{\alpha},
\end{aligned}
$$

где $\left\{Z_{N}\right\}_{N=1}^{\infty}$ - лобая последовательность фунтии такая, что при $s \rightarrow \infty$

$$
\left\|z_{N}-f^{(r+1, r+1)}\right\|_{\infty} \rightarrow 0
$$

будет асимптотически оптимальнои.

Локазательство. Нетрудно видеть, что ка кахдоя клетке $D_{i, N}$

$$
\left|f(u, y)-P\left(f, \Delta_{N}, x, y\right)\right|=\left|\iint_{D_{i, N}} f^{(r+1, r+1)}(u, v) K_{r}(x, u) K_{r}(y, v) d u d v\right| \text {. }
$$

Пусть $\left(\xi_{i}, \eta_{i}\right)$ - произвольвая точке ия $D_{i, N}$ и $A_{i}=f^{(r+1, r+1)}\left(\xi_{i}, \eta_{i}\right)$. IIR $x, y \in D_{i, N}$

$$
\left|f(x, y)-P\left(f, \Delta_{n}, x, y\right)\right|=\mid A_{i} \iint_{D_{i, N}} K_{r}(x, u) K_{r}(y, v) d u d v+
$$


$-75-$

$$
\begin{aligned}
& +\iint_{D_{i}, N}\left(f^{(r+1, r+1)}(u, v)-A_{i}\right) K_{r}(x, u) \mathcal{K}_{r}(y, v) d u d v \mid \geqslant \\
& \geqslant \max \left\{\left|A_{i}\right| h_{i}^{r+1} H_{i}^{r+1}|| \Theta r, k\left(\left(x-\alpha_{i}\right) / h_{i}\right)|| \Theta_{r, k}\left(\left(y-a_{i}\right) / H_{i}\right) \mid-\right. \\
& \left.\left.-x_{r, k}\left(\left(x-\alpha_{i}\right) h_{h_{i}}\right) x_{r, k}\left(\left(t_{i}-a_{i}\right) / H_{i}\right) \omega\left(f^{r+1, r+1)}, h_{i}^{2}+H_{i}^{2}\right)\right), 0\right\} .
\end{aligned}
$$

тогда надиётоя такое число $\lambda>0$, не эавися деe or $N$, что АЛя $i=1, N$

$$
\begin{aligned}
& \left\|f-P\left(f, \Delta_{N}\right)\right\|_{P\left(D_{i, N}\right)} \geqslant\left(h_{i} H_{i}\right)^{1 / \alpha}\left\|\Theta_{k}\right\|_{P}^{2} \times \\
& \times\left\|\max \left\{\left|A_{i}\right|-\lambda \omega\left(?^{(r+1, r+1)}, h_{i}^{2}+H_{i}^{2}\right), 0\right\}\right\|_{P} .
\end{aligned}
$$

Обозначим череs $g_{N}(x, y)$ Функцир, равнур

$$
\max \left\{\left|A_{i}\right|-\lambda \omega\left(f^{(r+1, r+1)}, h_{i}^{2}+H_{i}^{2}\right), 0\right\}
$$

AXA $x, y \in D_{i, N}(i=1, N)$.

月ено, что АлR $\mathrm{N} \rightarrow \infty$

$$
\iint_{D}\left|g_{N}(x, y)\right|^{\alpha} d x d y=\psi^{\alpha}(f)(1+o(1)) .
$$

Torá для $p \in[1, \infty)$

$$
\left\|f-P\left(f, \Delta_{N}\right)\right\|_{P}=\left\|\Theta_{r, k}\right\|_{P}^{2}\left(\sum_{i=1}^{N}\left\|g_{N}\right\|_{\alpha}^{p}\left(D_{i, N}\right)\right)^{1 / p} \text {. }
$$

Oт ода и из равенствв

$$
\min \left\{\sum_{k=1}^{n} C_{k}^{\alpha} \mid c_{k}>0: \sum_{k=1}^{n} c_{k}=c\right\}=c^{0} n^{1-\alpha},
$$

вери го тля $\alpha>1$ при $p \in[1, \infty)$, пс тучаеи

$$
\|f \cdot P(f, \Delta,)\|_{p} \geqslant\left\|\Theta_{r, k}\right\|_{r}^{2} N^{-r-1}\left\|g_{N}\right\|_{\alpha} \text {. }
$$


- $76-$

Отспда и из (II) сразу следует оценка для (8). Перепдём $x$ доказательству оценки сверху.

Пуств точка $\left(\xi_{i, j}, \eta_{i, j}\right) \in D_{i, j, j}$ такова, 10

$$
\begin{aligned}
& \iint_{D_{i, N}}\left(\left|f^{(r+1, r+1)}(x, y)\right|+\omega^{\gamma}\left(f^{(r+1, r+1)}, h_{i j}^{2}+H_{i, j}^{2}\right)\right)^{\alpha} d x d y= \\
& =h_{i, j} H_{i, j}\left(\left|f^{(r+1, r+1)}\left(\xi_{i, j i} \eta_{i, j}\right)\right|+\omega^{\gamma}\left(f^{(r+1, r+1)}, h_{i j}^{2}+H_{i, j}^{2}\right)\right)^{\alpha} . \\
& \left|f(x, y)-P\left(f, \Delta_{r}, x_{i} y\right)\right| \leq\left(H_{i, j} h_{i, j}\right)^{r+1}|| f^{(r+1, r+1)}\left(\xi_{i, j}, \eta_{i, j}\right) \mid \times \\
& \times \Theta_{r, k}\left(\left(x-\alpha_{i, j}\right) / h_{i, j}\right) \| \Theta_{r, k}\left(\left(y-a_{i, j}\right) / H_{i, j}\right) \mid+\omega\left(f^{(r+1, r+1)}, h_{i, j}^{2}+H_{i, j}^{2}\right) \times \\
& \left.\times \mathscr{x}_{r, k}\left(\left(x-\alpha_{i, j}\right) / h_{i, j}\right) \mathscr{x}_{r, k}\left(\left(y-a_{i, j}\right) / H_{i, j}\right)\right) .
\end{aligned}
$$

Пусть узль $x_{i, d}^{*}, y_{i, j}^{*}$ вибранв из условия (5) - (6), тогда из (5) получвек

$$
\int_{y_{j-1}^{*}}^{y_{i}^{*}} \omega^{d \gamma}\left(f^{(r+1, r+1)}, n^{-2}+m^{-2}\right) d y \leqslant \frac{1}{m} \psi_{N}^{\alpha} \text {. }
$$

Учитьвая, что при $N \rightarrow \infty$ будет $\Psi_{N} \rightarrow \Psi(f)$. получик

$$
H_{j}^{*} \leq \frac{2 \psi^{\alpha}(f)}{m} \omega^{-\alpha \gamma}\left(f^{(r+1, r+1)}, n^{-2}+m^{-2}\right)
$$

и, кроме того,

$$
H_{j}^{*} \geqslant \frac{\psi_{N}^{\alpha}}{m\left(M\left\|z_{N}\right\|_{\infty}\right)^{\alpha}},
$$

где $M>1$ такое, что $\left(M\left\|z_{N}\right\|_{\infty}\right)^{\alpha}>1$.

Тогда отсдда и из (6) еледует

$$
h_{i, j}^{*} \leqslant \frac{2 \psi^{\alpha}(f)}{h} \omega^{-\alpha \gamma}\left(f^{(r+1, r+1)}, h^{-2}+m^{-2}\right) \text {. }
$$

Отспдв и из свойств модуля непрерывности получвем

$$
\left(h_{i, j}^{*} H_{j}^{*}\right)^{r+1} \omega\left(f^{(r+1, r+1)},\left(h_{i, j}^{*}\right)^{2}+\left(H_{i, j}^{*}\right)^{2}\right) \leq 0\left(\frac{1}{N^{r+1}} \omega^{2 \gamma}\left(f^{(r+1, r+1)}, n^{-2}+m^{-i}\right)\right) .
$$


$-77-$

Tогда из (I3) имеем

$$
\begin{aligned}
& \left\|f-P\left(f, \Delta_{N}^{*}\right)\right\|_{p} \leq\left\|\Theta_{r, k}\right\|_{p}^{2}\left(\sum_{i, j}\left(H_{j}^{*} h_{i, j} \| f^{(r+1, r+1)}\left(\xi_{i, j}, \eta_{i, j}\right)\right)+\right. \\
& \left.\left.+\omega^{\gamma}\left(f^{(r+1, r+1)}, n^{-2}+m^{-2}\right)\right)^{\alpha / p / \alpha}\right)^{1 / p}+0\left(\frac{1}{N^{r+1}} \omega^{2 \gamma}\left(f^{(r+1, r+1)}, n^{-2}+m^{-2}\right)\right)= \\
& =\left\|, \eta_{r, k}\right\|_{F}^{2}\left(\sum_{i, j}\left(\iint_{D i, j, N}\left(\| f^{(r+1, r+1)}(x, y) \mid+w^{\gamma}\left(f^{(r+1, r+1)}, n^{-2}+m^{-2}\right)\right)^{\alpha}\right)^{p / \alpha}\right)^{1 / \rho}+ \\
& +O\left(\frac{1}{N^{r+4}} \omega^{2 \gamma}\left(f^{(r+1, r+1)}, n^{-2}+m^{-2}\right)\right)
\end{aligned}
$$

Отспда и ия неравенстве Кинковского немедленно получвем

$$
\begin{aligned}
& \left\|f-F\left(f, \Delta_{. j}^{*}\right)\right\|_{p} \leq\left\|\Theta_{r, k}\right\|_{P}^{2}\left(\sum _ { i , j } \left(\iint_{D_{i, j, N}}\left(\mid z_{N}(x, y) \|+\omega^{\gamma}\left(f^{r+1, r+}, n^{-2}+m^{-2}\right)\right)_{j}^{\alpha} d x d y+\right.\right. \\
& \left.\left.\left.+h_{i, j}^{*} H_{j}^{*} \omega^{2 \alpha \gamma / f^{(r+1, r+1)},-2}+m^{-2}\right)\right)^{p / \alpha}\right)^{1 / p}+0\left(\frac{1}{N^{r+1}} \omega^{2 \gamma}\left(f^{++1, r+1)}, n^{-2}, m^{-2}\right)\right) \leqslant \\
& \leq \frac{\left\|\Theta_{r, k}\right\|_{P}^{2}}{N^{r+1}} \Psi_{N}\left(1+\omega^{\gamma(2-\alpha)}\left(f^{(r+1, r+1)}, h^{2}+m^{-2}\right)\right)^{1 / \alpha} x \\
& \times\left(1+O\left(\omega^{2 \gamma}\left(f^{(r+1, r+1)}, h^{-2}+m^{-2}\right)\right)\right)
\end{aligned}
$$

Tах как при $N \rightarrow \infty \quad \Psi_{N} \rightarrow \Psi(f)$, то для $p \in[1, \infty)$ сразу следует оценка сверку.

Дая $p=\infty$ доказвтепьство проводится аналогично.

Биолиографи еские ссилки

I. B а p r a P. Тункциональюи внализ и теория вппроксимации в численнок анвлизе. М., I974.

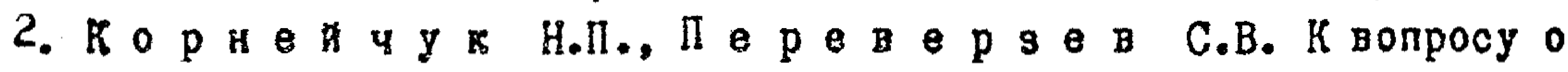
приближении Ф:нкции двух переменншх операторами, построеннкми на базе одномерных операторов // Теория функции и топология. Кнев., I983. 
- $78-$

3. І и ун А.А., С т ор ч а а В.Қ. 0 наилучшем ввборе узлов при приблихении функий локальными ормитоввми опнайнами // Уй. 1980. T. 32, 6 .

4. Iи Гу н А.А.. II у м дов при приблихении пинкций двумерными оплайами// Иоследования по совреме 'ным проблемам суммиронания и приближения функиия и: их прилохениям. Iнепропетровск, $і 982$.

JAK 5I7. 512

л.Г. Бодцун, А.И.Хальяова

ОБ АБСОЛЮТНОЯ СУМИРУЕАОГТИ ИНТ ЕТРАЛОВ ФУРЬЕ С

MHORUTEЛTVИ

I. Пусть фунхиия $f\left(u^{\prime}\right.$ интегрируема на кахдом конечном примехутке начиная о нуля и $S(1)=\int_{0}^{t} f(u) d u$. Пуоть

$$
z(y)=\int_{0}^{y} u(y, t) s(t) d t \text {. }
$$

Говорят, что $\int_{0}^{\infty} f(u) d u$ абоодотно оуммит, етоя рункииональным меruдом $T=(a(y, t))$ ихи оуммируен $|T|$, өсхи

$$
\int_{0}^{\infty}\left|\tau^{\prime}(y)\right| d y<\infty \text {. }
$$

Еоли

$$
a(y, t)=\left\{\begin{array}{cc}
\frac{P(y-t)}{P(y)}, & t \leq y, \\
0, & t>y,
\end{array}\right.
$$

где $P(y)=\int_{0}^{y} p(t) d t$. тогда ми имеөм дело о функционахьннм методом Г. Ф. Вороного [I]. Аналс. кчно, соли

$$
a(y, t)=\left\{\begin{array}{cc}
\frac{\alpha}{y}\left(1-\frac{t}{y}\right)^{\alpha-1}, & t \leq y, \\
0, & t>4,
\end{array}\right.
$$

тогда ми имеем дель о $(c, \alpha)$ me sодом, $\alpha>0$.

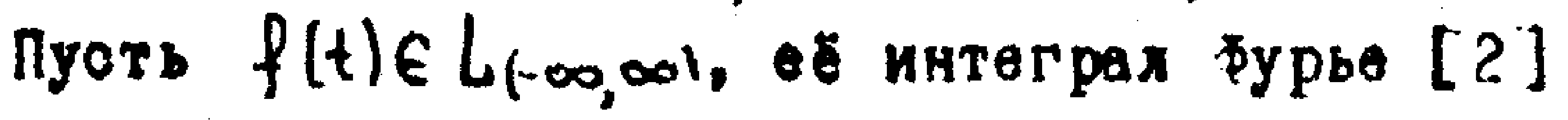

$$
f(1) \sim \int_{0}^{\infty}[a(y) \cos y t+h(y) \sin y t] d y=\int_{0}^{\infty} B\left(y_{1}\right) d y .
$$

